Proyecciones

Vol. $12 \mathrm{~N}^{0} 2$, pp.155-159 December 1993

Universidad Católica del Norte

Antofagasta - Chile

\title{
AN ORLICZ-PETTIS THEOREM FOR CONDITIONALLY CONVERGENT SERIES
}

\author{
CHRISTOPHER E. STUART
}

New Mexico State University, New Mexico, USA.

\begin{abstract}
in this note we define the idea of signed subseries convergent series in a normed linear space, and establish a result analogous to the Orlicz-Pettis theorem; namely, that weakly signed subseries convergent series are norm signed subseries convergent.
\end{abstract}




\section{Introduction.}

The classical Orlicz-Pettis theorem states that a weakly subseries convergent series in a normed vector space $X$ in subseries convergent with respect to the norm topology. In this note we show that weakly signed subseries convergent series (Definition 2.1) in $X$ are actually norm signed subseries convergent.

\section{Main Results.}

Definition 2.1. Let $X$ be a topological vector space, and $\left(x_{n}\right) \subseteq X$. A formal series $\sum x_{n}$ is signed subseries convergent if it satisfies:

a) for every subsequence $\left(x_{n_{k}}\right)$ of $\left(x_{n}\right)$ there is a choice signs $\left(s_{k}\right) \in\{-1,1\}^{N}$ sucli that $\sum_{k=1}^{\infty} s_{k} x_{n_{k}}$ converges, and

b) if $\sum_{k=1}^{\infty} s_{k} x_{n_{k}}$ converges, and $y_{l}=\sum_{k \in I_{l}} s_{k} x_{n_{k}}$, for $\left(I_{l}\right)$ and increasing sequence in $I_{0}$ (the set of all finite subintervals of $N$ ), there is a choice of signs $\left(t_{l}\right)$ such that $\sum_{l=1}^{\infty} t_{l} y_{l}$ converges. (Note that this implies $\sum y_{l}$ is also signed subseries convergent.)

By the following theorem of Dvoretsky and Hanani, every null sequence in a finite-dimensional normed space (i.e., $\mathbf{R}^{m}$ ) is signed subseries convergent:

Theorem 2.2. ([DH]) If $\left(x_{n}\right)$ is a null sequence in $\mathbf{R}^{m}$, then there is a choice of signs $\left(s_{n}\right)$ such that $\sum_{n=1}^{\infty} s_{n} x_{n}$ converges.

The conditions of Definition 2.1 are easily seen to be satisfied in this case, since any subsequence of a null sequence is null, so a) holds. If $\sum_{k=1}^{\infty} s_{k} x_{n_{k}}$ converges and $y_{l}=\sum_{k \in I_{l}} s_{k} x_{n_{k}}$ for $\left(I_{l}\right)$ increasing, then $\left(y_{l}\right)$ is a null sequence in $\mathbf{R}^{m}$. So by the Dvoretsky-Hanani theorem, there is a choice of signs $\left(t_{l}\right)$ such that $\sum_{l=1}^{\infty} t_{l} y_{l}$ converges, which is b).

Thus, the property of signed subseries convergence is in marked contrast to subseries convergence in finite-dimensional spaces, since subseries convergence implies absolute convergence in this case.

We now establish a result analogous to the Orlicz-Pettis theorem for signed subseries convergent series in a normed linear space $X$. The following theorem is a generalization of the Basic Matrix Theorem of Antosik and Mikusinski, and is used in the proofs of some preliminary results.

Theorem 2.3. (The signed Basic Matrix Theorem) Let $X$ be a Hausdorff topological group, and $x_{i j} \in X$ for $i, j \in \mathbf{N}$. Suppose

i) $\lim _{i} x_{i j}=x_{j}$ exists for each $j$ and 
ii) For each increasing sequence of positive integers $\left(m_{j}\right)$ there is a subsequence $\left(n_{j}\right)$ of $\left(m_{j}\right)$ and a cloice of signs $\left(s_{j}\right)$ such that $\left(\sum_{j=1}^{\infty} s_{j} x_{i n_{j}}\right)_{i=1}^{\infty}$ is Cauchy.

Then $\lim _{i} x_{i j}=x_{j}$ uniformly in $j$. In particular, $\lim _{i} x_{i i}=0$.

Proof. See ([S], Theorem 2, p. 96) for a proof of the Basic Matrix Theorem, or [St] for a proof the signed Basic Matrix Theorem, which is a straightforward generalization.

Definition 2.4. A sequence $\left(x_{n}\right)$ in a topological vector space $X$ is said to signed $\hat{N}$-convergent if for every subsequence of $\left(x_{n}\right)$ there is a further subsequence $\left(x_{n_{k}}\right)$ and a choice of signs $\left(s_{k}\right)$ such that $\sum_{k=1}^{\infty} s_{k} x_{n_{k}}$ converges.

Proposition 2.5. If $\left(x_{j}\right) \subseteq X$ is $\sigma\left(X, X^{\prime}\right)$ - signed- $\mathcal{K}$-convergent, then $\left\|x_{j}\right\| \longrightarrow 0$.

Proof. The proof is essentially that of [S], Proposition 16, p. 218, using Theorem 2.3 in place of the Basic Matrix Theorem.

Theorem 2.6. If $\left(x_{n}\right) \subset X$ is $\sigma\left(X, X^{\prime}\right)$-signed- $\mathcal{K}$-convergent, then $\left(x_{k}\right)$ is $\|\cdot\|$ signed- $\mathcal{K}$-convergent.

Proof. (The proof emulates that of [S], Theorem 19, p. 220).

By the proposition above, $\left(x_{k}\right)$ is norm convergent to 0 . Let $\left(y_{k}\right)$ be a subsequence of $\left(x_{k}\right)$ such that $\sum_{k=1}^{\infty}\left\|y_{k}\right\|<\infty$. Next, let $\left(z_{k}\right)$ be a subsequence of $\left(y_{k}\right)$ such that $\sum_{k=1}^{\infty} s_{k} z_{k}$ is weakly convergent to some $z \in X$ for some choice of signs $\left(s_{k}\right)$. The partial sums $S_{n}=\sum_{k=1}^{n} s_{k} z_{k}$ form a norm Cauchy sequence since the series $\sum\left\|z_{k}\right\|$ is absolutely convergent. Since the norm topology is linked to the weak topology, $S_{n} \longrightarrow z$ in norm (see [S], Definition 16.17). The argument can be applied to any subsequence of $\left(x_{k}\right)$, so $\left(x_{k}\right)$ is norm signed- $\mathcal{K}$-convergent.

We next prove a lemma that is a straightforward generalization of [S], Lemma 20, p. 221.

Lemma 2.7. Let $\sigma$ and $\tau$ be two vector topologies on the vector spaces $E$ with $\sigma$ linked to $\tau$. If every series $\sum_{k} x_{k}$ in $E$ wich is signed subseries convergent with respect to $\tau$ satisfies $\sigma-\lim x_{k}=0$, then every series in $E$ which is $\tau$-signed subseries convergent is also $\sigma$-signed subseries convergent.

Proof. If $\sum x_{n}$ is $\tau$-signed subseries convergent but fails condition a) in the definition for the $\sigma$-topology, then there exists a subseries $\sum_{k} x_{n_{k}}$ and a choice of sigus $\left(s_{k}\right)$ such that $\sum_{k} s_{k} x_{n_{k}}$ is $\tau$-convergent, but fails to be $\sigma$-Cauchy (using [S], Lemma 18,p. 219). Therefore, there exists an increasing sequence $\left(I_{m}\right)$ in $I_{0}$ and a $\sigma$-neighborhood of, $0, U$, such that

$$
\sum_{k \in I_{m}} s_{k} x_{n_{k}}=z_{m} \notin U \text {. }
$$


The series $\sum \tilde{z}_{m}$ is $\tau$-signed sulsseries convergent (using Definition $2.1 \mathrm{~b}$ )), so $\sigma-\lim z_{m}=0$, by hypothesis. This contradicts $z_{m} \notin U$.

A sinilar argument slows that condition b) must hold for the $\sigma$-topology. Indeed, if not, there exists a subsequence $\left(x_{n_{k}}\right)$ and an increasing sequence $\left(I_{k}\right) \subset$ $I_{0}$ such that the series $\sum s_{k} x_{n_{k}}$ is $\tau$-convergent for signs $\left(s_{k}\right)$ and $\sum t_{l} y_{l}$ converges with respect to $\tau$. where $y_{l}=\sum_{k \in I_{l}} s_{k} x_{n_{k}}$, but $\sum t_{l} y_{l}$ fails to be $\sigma$-Cauchy. Since $\sum y_{1}$ is $\tau$-signed subseries convergent, the arguinent in the preceding paragraph call be repeated to obtain a contradiction.

We can now prove the signed Orlicz-Pettis theoren.

Theorem 2.8. If $\sum x_{k}$ is $\sigma\left(X, X^{\prime}\right)$ signed subseries convergent in $\mathrm{X}$, then $\sum x_{k}$ is norm signed subseries convergent.

Proof. It follows from Proposition 2.5 that $\left\|x_{k}\right\| \longrightarrow 0$ because $\left(x_{k}\right)$ is $\sigma\left(X, X^{\prime}\right)$ signed $\mathcal{K}$-convergent. Since the nolm topology is linked to the weak topology, the theorem follows from the previous lemma.

We now state some open problems concerning signed subseries convergent series:

1) Recall that the summing basis of $c_{0}$ is the basis of vectors $x_{n}=\sum_{i=1}^{n} e_{i}$, where $\left(e_{i}\right)$ is the unit vector basis of $c_{0}$. It is well known that the summing basis is a conditional basis; indeed $\sum_{n=1}^{\infty} a_{n} x_{n}$ converges in $c_{0}$ if and only if $\left(a_{n}\right) \in c s$, the space of all convergent series (see [Si], Example 14.1). It follows easily, using the Dvoretsky-Hanani theorem, that the summing basis is a "signed subseries" basis. 'That is, $\sum_{n=1}^{\infty} a_{n} x_{n}$ is signed subseries convergent whenever the series converges. It would be interesting to have an example of a Banach space with no unconditional basis that has a signed subseries basis. In particular, does $C[0,1]$ have a signed subseries basis?

2) The equivalence of unconditional convergence, bounded multiplier convergence, and subseries convergence is well known in Banach spaces (see [S]). We say that a series $\sum_{n} x_{n}$ is signed unconditionally convergent if for every permutation $\pi: \mathbf{N} \longrightarrow \mathbf{N}$. there is a choice of signs $\left(s_{n}\right)$ such that $\sum_{n} s_{n} x_{\pi(n)}$ converges. Also, a series is signed bounded multiplier convergent if for every sequence $\left(a_{n}\right) \in l^{\infty}$ there is a choice of signs $\left(s_{n}\right)$ such that $\sum_{n} s_{n} a_{n} x_{n}$ converges. Are these properties equivalent in Banach spaces? It is easy to see that the summing basis is signed unconditional and signed bounded multiplier convergent.

3) Finally, is part b) in Definition 2.1 inplied by part a), or are they independent? 


\section{REFEREN CES}

[DH] Dvoretsky, A.; Hanani, C.: Sur les changements des signes d'une serie a termes complexes. C.R. Academie Des Sciences Paris, 225 (1947), 516518.

[S] Swartz, C.: An Introduction to Functional Analysis. Marcel Dekker, 1992.

[Si] Singer, I.: Bases in Banach Spaces I. Springer-Verlag New York, 1970.

[St] Stuart, C.: Weak sequential completeness of $\beta$-duals. Preprint.

Received: September 27, 1993.

Clıristopher E. Stuart

Departinent of Matliematical Sciences

New Mexico State University

Las Cruces, NM 88003, USA. 